\title{
Imaging of powerful terahertz beams
}

\author{
$\underline{\text { I. V. Andreev }}{ }^{1,2}$, V. M. Muravev ${ }^{1,2}$, A. R. Khisameeva ${ }^{1,2}$, G. E. Tsydynzhapov ${ }^{1,2}$, \\ I. V. Kukushkin ${ }^{1,2}$ \\ ${ }^{1}$ MWAVE LLC, Moscow, Russia, \\ ${ }^{2}$ Institute of Solid State Physics RAS, Chernogolovka, Russia, \\ ivan.andreev@terasense.com
}

$\mathrm{THz}$ frequency range is a field of constantly growing interest within modern applied physics. Nondestructive imaging, time-domain and frequency domain spectroscopy in $\mathrm{THz}$ range become important up-to-date techniques of material characterization [1]. Recently considerable interest was attracted by various $\mathrm{THz}$ personnel screening and security systems. Powerful $\mathrm{THz}$ sources, such as gyrotrons, could be used as a focused heating source for selective melting with controllable temperature distribution in glass and semiconductor industry [2], or for plasma heating into ITER tokamak. Lots of existing applications require quasi-optical setup, which demands proper beam alignment and focusing. Sometimes it becomes a complicated task since sub-THz radiation is invisible to the eye. We developed $\mathrm{THz}$ square cameras, which allows for real-time beam profiling of various vacuum-tube and semiconductor-based $\mathrm{THz}$ sources and could greatly simplify adjustment of quasi-optical setups. Cameras are suitable for both vacuum-tube (BWO, gyrotrons) and semiconductor-based (IMPATT and Gunn diodes) $\mathrm{THz}$ sources operating in range of 50-700 GHz.

Our group have developed a new semiconductorbased technology for detection of $\mathrm{THz}$ radiation in a broad range of 50-700 GHz. Detectors are fabricated from GaAs/AlGaAs quantum well heterostructures with high two-dimensional (2D) electron conductivity. Detection principle is rest on coupling of incident sub- $\mathrm{THz}$ radiation to so-called relativistic plasmons in 2D electron system [3] and subsequent rectification of plasmon ac potential on nonlinear defects, which provides a dc signal at the output of the detector. Single detector itself has a noise equivalent power (NEP) of $0.5 \mathrm{nW} / \mathrm{Hz}^{1 / 2}$ and ultrafast response time of $120 \mathrm{ps}$. GaAs detectors are fully compatible with common semiconductor processing lines, which makes them comparatively inexpensive, and allows to manufacture detectors on industrial scale and with reproducible properties.

Further to our research, we are developing a graphene-based detector in $\mathrm{THz}$ frequency range. Plasma oscillations in graphene have typical frequencies in $\mathrm{THz}$ range, which allows graphene to be used as a detector in this range of electromagnetic radiation. This material has the highest value or $2 \mathrm{D}$ electron mobility that provides high $2 \mathrm{D}$ conductivity and allows to observe weakly damped relativistic plasma oscillations. In contrast to common two-dimensional plasmons, the damping of relativistic plasma mode [3] is not determined by the inverse time of scattering of charge carriers. Thanks to weak damping, these plasma oscillations can be excited even at room tempera- ture when ordinary plasmons are strongly suppressed. Compared to existing analogs, the plasmon detector based on graphene should have the highest responsivity and fastest response time.

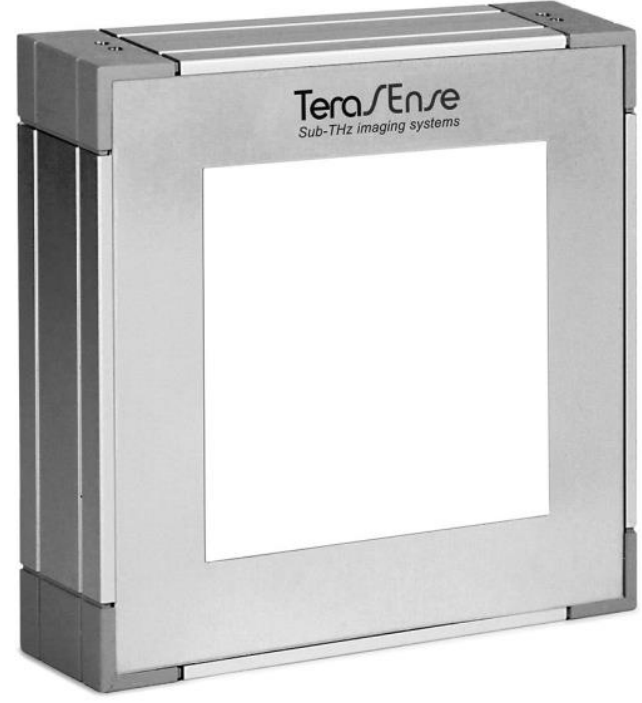

Fig. 1. Visual appearance of a Tera-4096 square imaging camera (64x64 pixels, 96x96 $\mathrm{mm}^{2}$ active area size).

Ownership of the detector technology allowed us to develop a line of $\mathrm{THz}$ imaging cameras (Fig. 1). Camera consists of a focal plane array of detectors (pixels), combined with amplification and readout circuitry. Cameras have a modular design, being assembled from blocks, which provides a very good scalability in pixel number and imaging area size. Most common camera sizes are 16x16, 32x32 and $64 \times 64$ pixels. High frame rate (up to $90 \mathrm{fps}$ ) makes possible to perform a real-time THz imaging. Typical camera parameters are summarized in the table below.

\begin{tabular}{|l|l|}
\hline Frequency range & $50-700 \mathrm{GHz}$ \\
\hline NEP & $1 \mathrm{nW} / \mathrm{Hz}^{1 / 2}$ \\
\hline Frame rate & $0.2-90 \mathrm{fps}$ \\
\hline Typical pixel number & $16 \times 16,32 \times 32,64 \times 64$ \\
\hline Pixel size & $1.5 \times 1.5$ or $3 \times 3 \mathrm{~mm}^{2}$ \\
\hline Features & $\begin{array}{l}\text { Mini-USB interface, TTL sync } \\
\text { out }\end{array}$ \\
\hline
\end{tabular}

As well as square cameras with frame rate not more than $100 \mathrm{fps}$, TeraSense also manufactures fast linear cameras with frame rate up to 5000 lines per second. Linear cameras are targeted at conveyor-belt based scanning applications.

TeraSense cameras proved itself as a flexible solution in a number of applications, such as nondestructive testing and quality control in industrial manufacturing lines [4], especially in area of humidity control in dry materials, mail inspection and security 
screening. Another promising application of $\mathrm{THz}$ imaging cameras is real-time beam profiling in various quasi-optical setups [5].

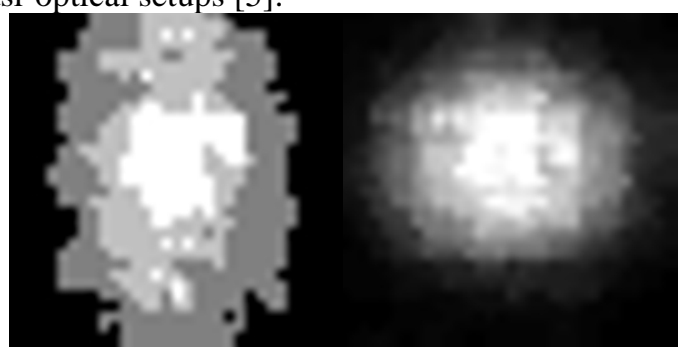

Fig. 2. Sample beam cross-sections of a $100 \mathrm{GHz}$ source, taken with Tera-1024 32x32 pixel camera for two different output geometries. Left: WR8 rectangular waveguide. Right: conical horn.

Square cameras could capture a cross-section power distribution of a beam less than in one second. This simplifies beam alignment procedure, makes possible to control angular pattern of the source, and solve other tasks arising in everyday life of experimental physicists and engineers working with quasioptical $\mathrm{THz}$ setups. Cameras are broadband sensitive in 50-700 GHz frequency range with several sensitivity peaks, which positions could be adjusted and optimized at the manufacturing stage. Minimal detectable power depends on the frame rate and is about $1 \mu \mathrm{W} / \mathrm{cm}^{2}$ at $1 \mathrm{fps}$, which allows cameras to operate even with low-power sources. Spatial resolution (discretization) of the beam cross-section image is limited by the pixel size and could be 1.5 or $3 \mathrm{~mm}$. Fig. 2 demonstrates sample beam cross-section images of the same source for various output geometries. Cameras could be used for alignment not only continuouswave, but pulsed beams as well. Operating with the pulsed beam, camera resembles mean beam power distribution. Camera pixels are tolerant to powerful beams, allowing to work not only with semiconductor-based sources, but with high-power vacuum-tube sources as well.

This work was partially financially supported by U.M.N.I.C. grant (contract No. 10667ГУ/2016).

\section{References}

1. Zhang, H., Sfarra, S., Saluja, K., Peeters, J., Fleuret, J., Duan, Y., Fernandes H., Avdelidis N., Ibarra-Castanedo $C$., Maldague, $X$. Non-destructive investigation of paintings on canvas by continuous wave terahertz imaging and flash thermography // Journal of Nondestructive Evaluation. 2017. V. 36, No. 2. P. 34.

2. Sklyarevich $V$., Shevelev M. Method for the rapid thermal treatment of glass and glass-like materials using microwave radiation. // U.S. Patent No. 6,408,649. 25 Jun. 2002.

3. Muravev, V. M., Gusikhin, P. A., Andreev, I. V., Kukushkin, $I$. $V$. Novel relativistic plasma excitations in a gated two-dimensional electron system // Physical review letters. 2015. V. 114. No. 10. P. 106805.

4. Zhang, H., Sfarra, S., Sarasini, F., Santulli, C., Fernandes, H., Avdelidis, N. P., Ibarra-Castanedo C., \& Maldague, $X$. $P$. Thermographic non-destructive evaluation for natural fiber-reinforced composite laminates // Applied Sciences. 2018. V. 8. No. 2. P. 240.

5. Yıldırım, I. O., Özkan, V. A., İdikut, F., Takan, T., Şahin, A. B., \& Altan, H. Characterization of a terahertz wave scanned imaging system for threat detection at standoff distances // Optical and Quantum Electronics. 2016. V. 48. No. 7. P. 367. 